\title{
The Policy Gap: A Survey of Patient-Perpetrated Sexual Harassment Policies for Residents and Fellows in Prominent US Hospitals
}

\author{
Elizabeth M. Viglianti, MD, MPH, MSc ${ }^{7}$, Andrea L. Oliverio, MD, MSc ${ }^{2}$, \\ Thomas M. Cascino, MD, MSc ${ }^{3}$, and Lisa M. Meeks, PhD ${ }^{4}$
}

'Department of Internal Medicine Division of Pulmonary and Critical Care, University of Michigan, Ann Arbor, MI, USA; ${ }^{2}$ Department of Internal Medicine Division of Nephrology, University of Michigan, Ann Arbor, MI, USA; ${ }^{3}$ Department of Internal Medicine Division of Cardiovascular Medicine, University of Michigan, Ann Arbor, MI, USA; ${ }^{4}$ Department of Family Medicine, University of Michigan, Ann Arbor, MI, USA.

J Gen Intern Med 34(11):2326-8

DOI: $10.1007 / \mathrm{s} 11606-019-05229-7$

(c) Society of General Internal Medicine 2019

$\mathrm{O}$ ne in five physicians has experienced sexual harassment by patients. ${ }^{1}$ The 2018 National Academy of Science, Engineering, and Medicine (NASEM) report on sexual harassment highlights the burden of patient-perpetrated sexual harassment, and calls for clear institutional policies to combat this in order to foster culture change. ${ }^{2}$

Despite the prevalence of patient-perpetrated sexual harassment and guidance from the NASEM report, it remains unknown to what extent graduate medical education (GME) training programs maintain policies and guidance that address patient-perpetrated sexual harassment.

\section{METHODS}

Following IRB approval, we conducted a web-based survey of designated institutional officials (DIO) at the top 20 US hospitals as identified by US News and World Report "Best Hospital" rankings in 2017. ${ }^{3}$ Weekly reminder emails were sent for one month with subsequent letters and phone calls (maximum of three telephone calls) to nonresponding DIOs. Two hospitals shared a DIO, resulting in a sample size of 19 institutions.

Drawing on the UK General Medical Council and the American Medical Association definitions, we operationalized sexual harassment as "unwelcomed attention or behavior of a sexual nature that might be offensive or cause a person to feel unsafe and uncomfortable." ${ }^{4}$ Officials were asked whether they (1) maintained a general sexual harassment policy; (2) offered training for responding to sexual harassment; (3)

$\overline{\text { Prior Presentations Results were shared as a poster presentation at the }}$ American Thoracic Society International Conference meeting in May 2019 in Dallas, TX.

Published online August 14, 2019 maintained a policy addressing patient-perpetrated sexual harassment; (4) offered training for responding to patientperpetrated sexual harassment; (5) had a protocol for reporting patient-perpetrated sexual harassment; and (6) if patientperpetrated sexual harassment was a problem in their GME programs.

In addition, two investigators (EMV and ALO) independently reviewed all submitted and publicly available GME policies for each hospital. Differences in review of the policies were discussed until the investigators reached consensus.

\section{RESULTS}

The DIOs surveyed oversee approximately 25,000 residents and fellows. Of the 19 DIOs contacted, 14 responded to question one and endorsed having a sexual harassment policy. Eleven completed the survey. Six of the 11 (54.5\%) responders reported providing sexual harassment training, while only three of the $11(27.3 \%)$ reported policies on patientperpetrated sexual harassment with associated training. Six of the $11(54.5 \%)$ responders reported patient-perpetrated sexual harassment was a problem at their GME program (Fig. 1a).

Sexual harassment policies were available online for 18 institutions and were independently reviewed. All 18 policies defined sexual harassment consistent with our definition. Ten programs maintained policies that implied the inclusion of patient-perpetrated sexual harassment in their definition, of which six policies specifically addressed patient-perpetrated sexual harassment. Only four of the policies included a reporting process within the policy. Of those, only one provided clear guidance on how to effectively address the behavior and clinical needs of the patient (Fig. 1b).

\section{DISCUSSION}

Patient-perpetrated sexual harassment is an identified issue for residents and fellows ${ }^{4}$ and contributes to physician burnout. ${ }^{5}$ Despite this, only six of the 18 institutions whose policies were 
a

Online Review of Sexual Harassment Policies

$\mathrm{N}=18$

Sexual harassment policy identified

Patient-perpetrated sexual harassment policy identified

Protocol for specifically reporting patient-perpetrated sexual harassment

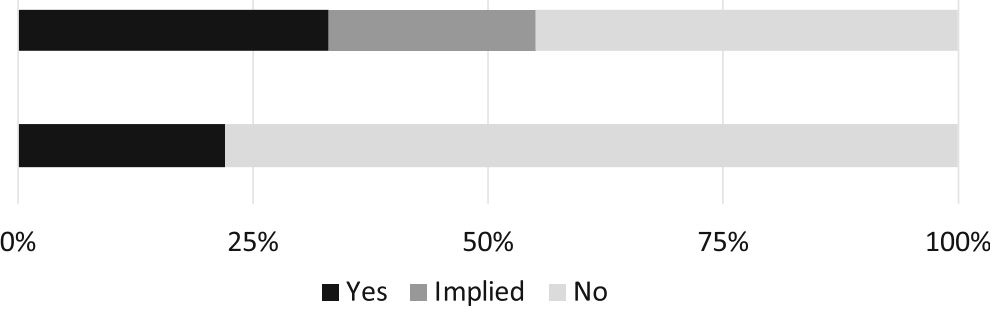

b

\section{Reported Sexual Harassment Policies \\ $\mathrm{N}=11$}

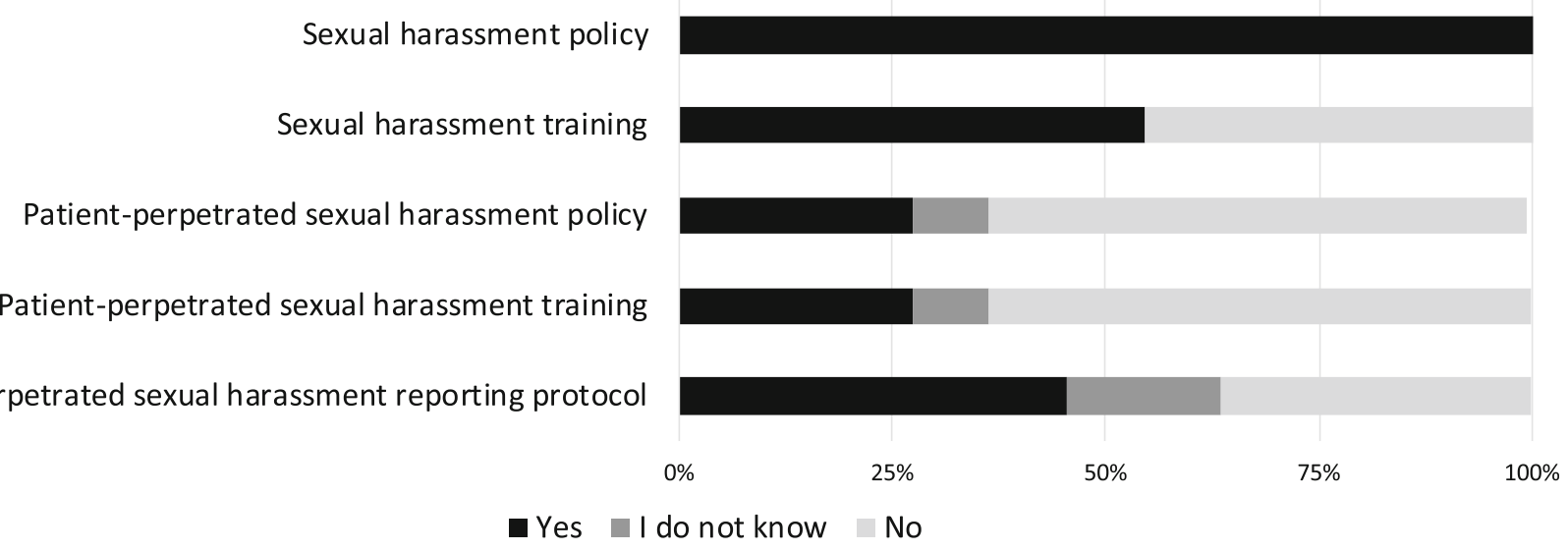

Figure 1 Sexual harassment and patient-perpetrated sexual harassment policies and protocols. a The presence of sexual harassment and patient-perpetrated sexual harassment policies, training, and protocols as reported by DIOs. b Review of sexual harassment and patientperpetrated sexual harassment policies and protocols. The label "implied" refers to policies in which the harasser was not considered a supervisor or colleague but a third party or visitor, and "patient" was never directly mentioned.

reviewed online had specifically addressed patient-perpetrated sexual harassment and only one included direction for reporting the patient while continuing clinical care. Patientperpetrated sexual harassment warrants distinct attention. Residents and fellows are at increased risk for victimization given their unique positioning between learner and employee and significant workloads. The NASEM report advises institutions to proactively address sexual harassment, including patientperpetrated events, through easily accessible guidance and response training. ${ }^{2}$ Yet our survey and analysis suggest an absence of such policy within the "Top 20 Hospitals".

Limitations of our analysis include small sample size and descriptive data. Our findings may not be generalizable to all hospitals and GME programs. We did not account for sexual harassment training mandated under individual state laws, nor any reporting mechanisms detailed outside of online policies. Further investigation that broadens the analysis to all GME programs and identifies key policies and cultural environments associated with decreased incidence of sexual harassment is needed.

Patient-perpetrated sexual harassment can result in destabilization, isolation, and emotional turmoil for residents and fellows. ${ }^{5}$ While institutions cannot prevent inappropriate patient behavior, they can set expectations that contribute to institutional culture. Hospitals can send a clear message to all stakeholders: we will not tolerate abuse in any form. Empowering residents, fellows, and their supervisors to recognize and respond to patient-perpetrated sexual harassment through policy and training may mitigate future harms, increase physician retention, and improve wellbeing. 


\section{Acknowledgments:}

The authors wish to thank Dr. Theodore J. Iwashyna for his continued support.

Funding/Support: This work was financially supported by $\mathrm{NIH}$ grants T32 HL7749-25 (EMV), T32 DKOO7378-38 (ALO), and T32HLOO7853 (TMC).

Corresponding Author: Elizabeth M. Viglianti, MD, MPH, MSc: Department of Internal Medicine Division of Pulmonary and Critical Care, University of Michigan, 2800 Plymouth Road, NCRC Building 16 373C, Ann Arbor, MI 48109, USA (e-mail: eviglian@med.umich.edu). Compliance with Ethical Standards:

Conflict of Interest: The authors declare that they do not have a conflict of interest.

\section{REFERENCES}

1. Fnais N, Soobiah C, Chen MH, Lillie E, Perrier L, Tashkhandi M, Straus SE, Mamdani M, Al-Omran M, Tricco AC. Harassment and discrimination in medical training: a systematic review and meta-analysis. Acad Med 2014; 89: 817-27.

2. The National Academies of Sciences, Engineering, and Medicine. Sexual Harassment of Women: Climate, Culture, and Consequences in Academic Sciences, Engineering, and Medicine. Washington, DC: The National Academies Press; 2018.

3. The U.S. News and World Report. US. U.S news announces $2017-18$ best hospitals. Available at: https://www.usnews.com/info/blogs/press-room/ articles/2017-08-08/us-news-announces-2017-18-best-hospitals. Accessed June 25, 2019.

4. Viglianti EM, Oliverio AL, Meeks LM. Sexual harassment and abuse: when the patient is the perpetrator. Lancet 2018; 392: 368-70.

5. Dzau VJ, Johnson PA. Ending Sexual Harassment in Academic Medicine. N Engl J Med 2018;379:1589-91

Publisher's Note Springer Nature remains neutral with regard to jurisdictional claims in published maps and institutional affiliations. 